\title{
Reservorios de biomasa y carbono en el arbolado de la primera sección del Bosque de Chapultepec, Ciudad de México
}

\section{Biomass and carbon storage in trees of the first section of Chapultepec Park, Mexico City}

\author{
Stephanie Fabiola López-López', Tomás Martínez-Trinidad ${ }^{1 *}$, Héctor Mario Benavides-Meza², \\ Moisés García-Nieto' y Gregorio Ángeles-Pérez'
}

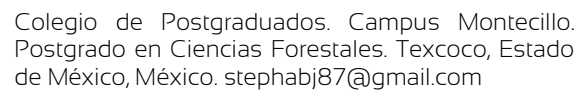

\author{
2 Intituto Nacional de Investigaciones Forestales \\ Agrícolas y Pecuarias. Centro Nacional de Investi- \\ gación Disciplinaria en Conservación y Mejora- \\ miento de Ecosistemas Forestales. Ciudad de \\ México, México \\ * Autor de correspondencia. tomtz@acolpos.mx

\footnotetext{
e
}

\section{RESUMEN}

Durante los últimos años ha habido un creciente interés por cuantificar los reservorios de carbono en los bosques naturales y urbanos y su potencial en la mitigación del cambio climático. En el presente estudio se estimaron los depósitos de biomasa y C aéreos del arbolado de la primera sección del Bosque de Chapultepec, en la Ciudad de México y se analizó su relación con el área basal y la composición de especies. Los resultados del inventario indican que el reservorio de $\mathrm{C}$ en el área verde de estudio es importante, al menos con respecto a la cantidad de $\mathrm{CO}_{2}$ que se emite de manera local. Además, para la especie dominante, Fraxinus ubdei, se compararon las estimaciones de biomasa de ecuaciones generadas en bosques naturales y urbanos con las obtenidas en siete árboles del sitio; la ecuación de ambientes urbanos generó una mejor estimación. A partir del inventario correspondiente a $27.3 \%$ de las áreas verdes de la primera sección y de la aplicación de relaciones alométricas, se estimaron reservorios totales de 24217 Mg de biomasa y $11226 \mathrm{Mg}$ de C. La composición de especies y el área basal influyeron en los depósitos de biomasa y C. El valor medio de contenido de C es alto en comparación con los registrados en bosques urbanos de otras ciudades del mundo, por lo que el Bosque de Chapultepec puede ser considerado un importante reservorio.

PALABRAS CLAVE: bosque urbano, captura de $\mathrm{CO}_{2}$, estructura arbórea, Fraxinus uhdei, servicios ambientales.

\begin{abstract}
In recent years, there has been a growing interest in quantifying carbon stocks in urban forests in order to assess their potential in climate change mitigation. In the present study the aboveground biomass and C pools were estimated at the first section of the Chapultepec Park and its relationship with the basal area and species composition was analyzed. Carbon stock of this green area is expected to be important, at least with respect to local neighborhood's emissions of $\mathrm{CO}_{2}$. For Fraximus uhdei, the dominant species, biomass estimates generated in natural and urban equations were compared with those obtained in seven trees sampled at the site; the urban equation produced the best estimates. Based on inventories of $27.3 \%$ of the green areas of the first section and the use of allometric relationships, the total reservoir was estimated at $24217 \mathrm{Mg}$ of biomass and $11226 \mathrm{Mg}$ of $\mathrm{C}$. The species composition and basal area significantly influenced biomass and $\mathrm{C}$ pools. The average $\mathrm{C}$ content was relatively high compared with those indicated in urban forests in other cities in the world, thus Chapultepec Park can be considered an important reservoir.
\end{abstract}

KEYWORDS: urban forest, $\mathrm{CO}_{2}$ capture, vegetation structure, Fraxinus uhdei, ecosystem services. 


\section{INTRODUCCIÓN}

Los bosques urbanos están conformados principalmente por el arbolado de alineación y el ubicado en áreas verdes en los límites de las ciudades (Benavides-Meza, 1989). El componente arbóreo es uno de los más importantes del ecosistema urbano en comparación con otros elementos del paisaje, ya que aporta beneficios económicos y sociales tales como: recreación, atenuación del ruido, reducción de gastos de atención médica, ahorros en el consumo de energía, mejora de la salud mental, incremento en el valor de viviendas, entre otros (Benavides-Meza, 1989; Akbari, Davis, Dorsano, Huang y Winnett, 1992; Harris, Clark y Matheny, 2004; Livesley, McPherson y Calfapietra, 2016). Además, el bosque urbano provee diversos servicios ecosistémicos, entre los que destaca la remoción de contaminantes atmosféricos, la fijación de carbono, regulación y modificación del microclima, captación de agua de lluvia y control de escorrentías, contribuyendo así a mejorar la calidad de vida de sus habitantes (Akbari et al., 1992; Heisler, 1986; Harris et al., 2004; Livesley et al., 2016).

Un servicio que adquirió gran relevancia por la necesidad de mitigar los efectos del calentamiento global es la captura de $\mathrm{C}\left(\mathrm{CO}_{2}\right)$ por las plantas y su transformación a biomasa (McPherson y Simpson, 1999; Rügnitz-Tito, Chacón-León y Porro, 2008; Liu y Li, 2012). Además, los árboles en paisajes urbanos aportan humedad al ambiente y, cuando están en la ubicación adecuada, regulan la incidencia de energía solar y la velocidad del viento; esto resulta en una modulación de la temperatura del microclima, que produce ahorro del consumo de electricidad para calentar o enfriar edificaciones (Nowak, 1994; McHale, McPherson y Burke, 2007). Por lo tanto, los árboles son depósitos naturales de $\mathrm{C}$ y contribuyen a reducir las emisiones de gases de efecto invernadero relacionadas con la generación de energía (Nowak, 1994; McPherson y Simpson, 1999).

$\mathrm{El}$ interés por cuantificar los reservorios de $\mathrm{C}$ en los bosques urbanos es creciente, con el fin de valorar su potencial en la mitigación en la emisión de los gases de efecto invernadero y así reducir su efecto en el cambio climático (McHale et al. 2007; Timilsina, Escobedo,
Straudhammer y Brandeis, 2014). Estas estimaciones se basan en ecuaciones de volumen, biomasa o carbono o ambos, en función de el diámetro y la altura total del árbol (Nowak, 1994; McHale et al. 2007), o mediante imágenes obtenidas a través de percepción remota (Liu y Li, 2012). Los bosques urbanos conforman depósitos abundantes de C y contribuyen al proceso de mitigación, ya que capturan $\mathrm{CO}_{2}$ y lo fijan como biomasa (McPherson y Simpson, 1999; Aguaron y McPherson, 2012). Por ejemplo, Liu y Li (2012) estimaron que el $\mathrm{C}$ almacenado en arbolado ubicado dentro del tercer anillo vial de la ciudad de Shenyang (3.5\% de la superficie total) equivale a $3.02 \%$ de las emisiones por combustibles fósiles anuales y la captura puede mitigar $0.26 \%$ de las emisiones totales anuales. Nowak, Greenfield, Hoehn y Lapoint (2013) cuantificaron un reservorio de 643 millones de megagramos en el bosque urbano de los Estados Unidos, con base en datos de 28 ciudades y seis estados.

En México, hay pocos estudios sobre los reservorios y la captura de $\mathrm{C}$ en las áreas verdes urbanas y no se ha cuantificado su contribución dentro de las acciones de mitigación de las ciudades (Velasco-Rodríguez et al. 2014). La disponibilidad de ecuaciones alométricas para todas las especies plantadas es una de las limitantes para la estimación precisa de $\mathrm{C}$ en los bosques urbanos (McPherson y Simpson, 1999). Al emplear ecuaciones de tipo general, se debe considerar que estas se hayan desarrollado en condiciones edafoclimáticas similares al sitio de estudio, con árboles de dimensiones semejantes e incluso se validen con datos de biomasa de un conjunto de árboles del área obtenidos por el método destructivo (Rügnitz et al. 2008), o a través de modelos alométricos desarrollados a partir de la medición de las dimensiones de algunas ramas y la determinación de su biomasa, o mediante índices estructurales de la copa basados en la teoría fractal que son de utilidad para describir la arquitectura de los árboles (no destructivos) (Dobbs, Hernández y Escobedo, 2011; López-López, Martínez-Trinidad, Benavidez-Meza, García-Nieto, y de los Santos-Posadas, 2017).

La cuantificación de reservorios de arbolado urbano con ecuaciones derivadas en ambientes naturales debe 
ajustarse porque las condiciones ambientales para el desarrollo de los árboles son diferentes en las ciudades (McPherson y Simpson, 1999; McHale, Burke, Lefsky, Pepper, y McPherson, 2009). En virtud de que se han registrado sobre o subestimaciones significativas en la biomasa de árboles urbanos al utilizar ecuaciones de bosque naturales, es recomendable usar modelos generados con árboles urbanos para obtener estimaciones más precisas (Nowak, 1994; McHale et al. 2009).

Para incrementar la capacidad de mitigación del calentamiento global deberá conocerse cómo la estructura y la composición de especies del arbolado urbano influyen en su potencial para capturar y almacenar $\mathrm{CO}_{2}$ (Nowak, Stevens, Sisinni y Luley, 2002; Escobedo, Varela, Zhao, Wagner y Zipperer, 2010; Timilsina et al. 2014). Sin embargo, aún se requieren estudios para conocer el potencial de reserva de los bosques urbanos que coadyuven a definir estrategias de manejo que contribuyan a la reducción de gases de efecto invernadero en la atmósfera. Por ejemplo, la contabilización del potencial de captura de C en bosques urbanos como el de Chapultepec (686.01 ha) y su relación con la estructura y composición de la masa arbórea permitirá estructurar un manejo que favorezca la captura y almacenamiento de $\mathrm{C}$ en zonas urbanas similares o aledañas. Además, la cuantificación de los servicios ambientales que provee el arbolado urbano, contribuye a valorarlo en términos tangibles y sustentar políticas públicas que promuevan su mantenimiento, así como la apertura de nuevos espacios con vegetación.

\section{OBJETIVOS}

El objetivo del presente estudio fue cuantificar el C aéreo almacenado en el arbolado de la primera sección del Bosque de Chapultepec, ya que, por su extensión, se espera que el reservorio sea representativo respecto a la cantidad de $\mathrm{CO}_{2}$ emitido de manera local. Además, se propuso comparar la estimación de biomasa aérea para la especie dominante a través de ecuaciones alométricas desarrolladas, una en ambientes naturales donde se distribuye la especie y otra en ambientes urbanos, con la hipótesis de que el segundo modelo proporcionará una mejor estimación de la biomasa.

\section{MATERIALES Y MÉTODOS}

\section{Área de estudio}

La primera sección del Bosque de Chapultepec está situada en la delegación Miguel Hidalgo de la Ciudad de México y abarca 274.08 ha a una altitud entre $2240 \mathrm{~m}$ y $2280 \mathrm{~m}$; presenta un clima templado subhúmedo con lluvias en verano (Programa Universitario de Estudios sobre la Ciudad [PUEC], 2002; Gobierno del Distrito Federal [GDF], 2006). Con base en un muestreo sistemático, se estima que el arbolado de esta sección está conformado por cerca de 55000 ejemplares pertenecientes a 110 especies arbóreas, de las cuales Ligustrum lucidum W.T. Aiton, Fraxinus ubdei (Wenz.) Lingelsh. y Hesperocyparis lusitanica (Mill.) Bartel son las más abundantes (PUEC, 2002; GDF, 2006).

\section{Muestreo e inventario del arbolado}

Se obtuvieron datos derivados de inventarios independientes del arbolado de la primera sección, efectuados por la Comisión Nacional Forestal (CONAFOR) y el Instituto Nacional de Investigaciones Forestales Agrícolas y Pecuarias (INIFAP) entre 2008 y 2014. En virtud de lo anterior, se aplicó un muestreo por conglomerados unietápico, en el que se definieron 37 conglomerados a través de una zonificación operativa del área de estudio (áreas de carácter técnico-administrativas para su manejo). Se seleccionaron al azar 11 zonas operativas (conglomerados) que contaban con un censo de su arbolado, las cuales congregaron una superficie de 49.73 ha y representaron $27.32 \%$ del total de área verde (182 ha). Los conglomerados incluían tanto bosquetes como arbolado de alineación con diferente densidad, por lo que se consideraron representativos (Fig. 1).

En los inventarios del Inifap y la Conafor se midieron los árboles con diámetro a la altura del pecho (DAP) mayor a cinco cm (Pearson, Brown y Birdsey, 2007). El número de individuos de cada taxón se determinó a partir de los registros de especie por conglomerado y con el DAP se calculó el área basal (AB) para determinar su densidad (Ecuación 1). 


$$
A B=\frac{\pi}{4} D A P^{2}
$$

La densidad y dominancia relativa de las especies se determinaron para calcular el índice de valor de importancia
(IVI) y su valor relativo (IVIR $=$ IVI $/ 2)$, con el fin de jerarquizar su dominancia (Ecuaciones 2 a 6) (LozadaDávila, 2010; Zarco-Espinosa, Valdez-Hernández, Ángeles-Pérez y Castillo-Acosta, 2010):

Índice de importancia $=$ Densidad relativa $(\%)+$ Dominancia relativa $(\%)$

$$
\text { Densidad relativa }(\%)=\frac{\text { Densidad absoluta de cada especie }}{\text { Densidad absoluta todas las especies }} * 100
$$

donde:

$$
\text { Densidad absoluta }=\frac{\text { Número de individuos de la especie i }}{\text { Área muestreada }}
$$

$$
\text { Dominancia relativa }(\%)=\frac{\text { Dominancia absoluta de cada especie }}{\text { Dominancia absoluta todas las especies }} * 100
$$

donde:

$$
\text { Dominancia absoluta }=\frac{\mathrm{AB} \text { de la especie } \mathrm{i}}{\text { Área muestreada }}
$$

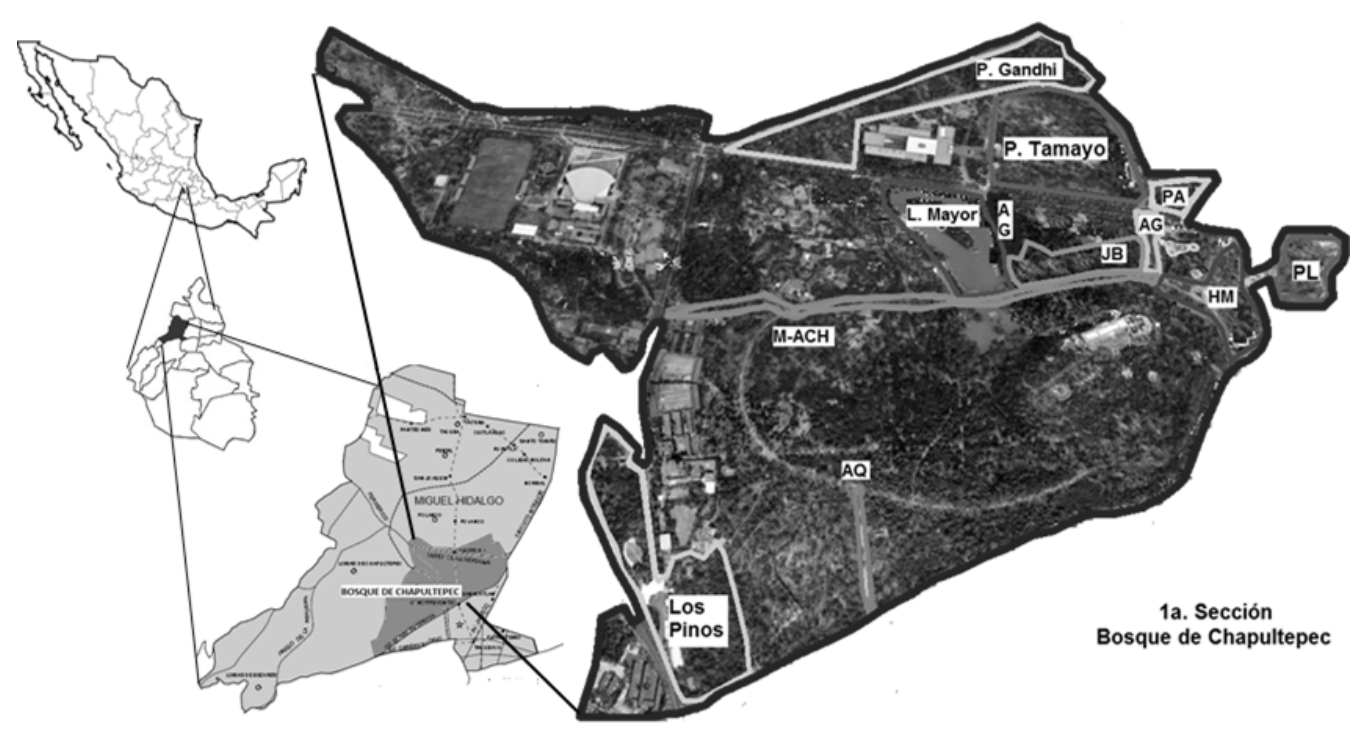

FIGURA 1. Zonificación de la superficie muestreada en el área de estudio. $\mathrm{AQ}=$ Acceso Quebradora, M-ACH = Milla-Acceso Chivatito, AG = Acceso Gandhi-Grutas, JB = Jardín Botánico, $\mathrm{PA}=$ Parque La Amistad, HM = Histórico Monumental y PL = Puerta de Leones. 


\section{Estimación del reservorio}

Para cuantificar la biomasa aérea de las especies registradas se efectuó la revisión bibliográfica de ecuaciones alométricas. En 13 taxones se emplearon fórmulas de volumen de árboles urbanos y se aplicaron factores de conversión a peso fresco (Tabla 1), así como una constante de 0.56 para calcular el peso seco (Pillsbury, Reimer y Thompson, 1998; McPherson, Simpson, Peper y Aguaron, 2008; McHale et al. 2009). Se utilizaron las ecuaciones propuestas por Jenkins, Chojnacky, Heath y Birdsey (2003), que incluyen varias especies presentes en el área de estudio, entre estas la fórmula “mixed hardwood” que se empleó en los individuos muertos, con una reducción de 3\% o 15\% por la ausencia de follaje o de ramas, respectivamente. Esta reducción se relaciona con el peso seco que por lo general aportan esos componentes del árbol (Pearson et al. 2007) (Material suplementario).

TABLA 1. Número de individuos, densidad, dominancia e IVIR de las especies presentes en el arbolado de 49.73 ha del área de estudio.

\begin{tabular}{lccccc}
\hline \multicolumn{1}{c}{ Especie } & $\begin{array}{c}\text { Número de } \\
\text { individuos }\end{array}$ & $\begin{array}{c}\text { Densidad } \\
\text { relativa (\%) }\end{array}$ & $\begin{array}{c}\text { Área basal } \\
\left(\mathrm{m}^{2}\right)\end{array}$ & $\begin{array}{c}\text { Dominancia } \\
\text { relativa (\%) }\end{array}$ & IVIR (\%) \\
\hline Fraxinus uhdei & 3615 & 25.42 & 369.75 & 26.47 & 25.95 \\
Ligustrum lucidum & 3735 & 26.26 & 336.87 & 24.12 & 25.19 \\
Hesperocyparis lusitanica & 2384 & 16.76 & 118.28 & 8.47 & 12.62 \\
Taxodium mucronatum & 337 & 2.37 & 114.78 & 8.22 & 5.29 \\
Eucalyptus globulus & 158 & 1.11 & 95.14 & 6.81 & 3.96 \\
Pinus sp. & 706 & 4.96 & 26.78 & 1.92 & 3.44 \\
Eucalyptus camaldulensis & 380 & 2.67 & 45.89 & 3.29 & 2.98 \\
Casuarina equisetifolia & 442 & 3.11 & 31.80 & 2.28 & 2.69 \\
Phoenix canariensis & 122 & 0.86 & 55.03 & 3.94 & 2.40 \\
Jacaranda mimosifolia & 324 & 2.28 & 27.38 & 1.96 & 2.12 \\
Liquidambar styraciflua & 227 & 1.60 & 11.96 & 0.86 & 1.23 \\
Eucalyptus sp. & 149 & 1.05 & 18.59 & 1.33 & 1.19 \\
Schinus molle & 43 & 0.30 & 21.95 & 1.57 & 0.94 \\
Otras (89) & 1601 & 11.26 & 122.42 & 8.77 & 10.01 \\
\hline Total & 1423 & 100 & 1396.64 & 100.00 & 100.00 \\
\hline
\end{tabular}

Siete individuos de F. uhdei se seleccionaron al azar a partir de un listado de árboles del sitio de estudio, en proporción a la cantidad de ejemplares registrados en cuatro categorías diamétricas definidas entre $5 \mathrm{~cm}$ y $80 \mathrm{~cm}$ de DAP. Los individuos seleccionados (12.5 cm y $69.0 \mathrm{~cm}$ de DAP) se cosecharon para cuantificar su biomasa, se seccionaron en 
sus diferentes compartimientos (tronco, ramas y follaje) y se pesaron en una báscula con capacidad de $6 \mathrm{~kg}$ y resolución de $0.2 \mathrm{~g}$. En los árboles mayores a $30 \mathrm{~cm}$ de DAP, el volumen del tronco se estimó a través de la cubicación de las trozas mediante la fórmula de Smalian (Ecuación 7) y la densidad se determinó a partir de cuatro secciones de madera de cada ejemplar.

$$
\text { Volumen }=\frac{S_{1}+S_{2}}{2} L
$$

donde:

$S_{1}=$ superficie de la sección inicial

$S_{2}=$ superficie de la sección final

$L=$ longitud de la troza

De cada individuo se tomaron siete muestras de follaje y cuatro de madera de tronco y ramas, para secarlas en una estufa a $70{ }^{\circ} \mathrm{C}$ por $48 \mathrm{~h}$ o hasta obtener peso constante y calcular el peso seco. La relación peso fresco: peso seco de cada compartimento del árbol se utilizó para estimar la biomasa y la suma del peso seco de todos los componentes constituyó la biomasa total de cada ejemplar (AcostaMireles, Vargas-Hernández, Velázquez-Martínez y Etchevers-Barra, 2002). Los valores de biomasa se compararon con las estimaciones de una ecuación generada en bosques naturales (Ares y Fownes, 2000) y otra en áreas verdes urbanas por (Pillsbury et al. 1998). La ecuación con la mejor aproximación se eligió para efectuar la estimación del reservorio de esta especie.

Para la cuantificación del $\mathrm{C}$ se emplearon relaciones alométricas (Material suplementario), o una vez determinada la biomasa se le aplicó el factor de conversión de 0.47 recomendado por el Intergovernmental Panel on Climate Change [IPCC] (2006). Para F. ubdei se utilizó un valor de 0.45 , que fue la proporción media de contenido de $C$ obtenida de 24 muestras de tronco, ramas y follaje de los ejemplares cosechados, analizadas con el determinador Shimadzu A500 en el Laboratorio de Química Ambiental del Colegio de Postgraduados.
Los reservorios de biomasa y $\mathrm{C}$ aéreos se estimaron por especie y zona inventariada y este último se multiplicó por la constante de conversión de 3.67 para conocer la porción de carbono [3.67 es la porción de C en la molécula de $\mathrm{CO}_{2}$ ] (McPherson y Simpson, 1999). Para definir si las características físicas (porte y talla) de las especies tienen repercusiones en el almacén, se realizó un análisis de correlación de Pearson entre el índice de IVIR y el aporte de $\mathrm{C}$ de cada taxón.

A partir de los contenidos de biomasa y $\mathrm{C}$ aéreos $(\mathrm{Mg}$ $\mathrm{ha}^{-1}$ ) registrados en los 11 conglomerados, se estimaron las medias y desviaciones estándar a través de un estimador de razón, debido a que los conglomerados eran de tamaño desigual (Ecuaciones 8 y 9).

$$
\text { Estimador de la media }(\overline{\mathrm{X}})=\hat{R}=\frac{\sum_{i}^{n} X_{i}}{\sum_{i}^{n} M_{i}}
$$

donde:

$X_{\mathrm{i}}=$ valor de la característica $\mathrm{X}$ en el conglomerado $i$ de la muestra

$M_{i}=$ número de unidades elementales (individuos) en el conglomerado $i$

$n=$ número de conglomerados en la muestra

$$
\hat{D} \hat{E}(X)=\sqrt{\frac{1-f}{n M^{2}}\left(\sum_{i}^{n} X_{i}^{2}+\hat{R}^{2} \sum_{i}^{n} M_{i}^{2}-2 \hat{R} \sum_{i}^{n} X_{i} M_{i}\right)}
$$

donde:

$\mathrm{f}=\quad$ la fracción muestral $\mathrm{n} / \mathrm{N}$

$\mathrm{n} \bar{M}^{2}=$ número total de unidades elementales en la muestra

$\mathrm{X}_{\mathrm{i}}=\quad$ valor de la característica $\mathrm{X}$ en el conglomerado $i$

$\hat{R}=$ estimador de la media (estimador de razón)

$\mathrm{M}_{\mathrm{i}}=$ unidades elementales en el conglomerado $i$

Los totales de los reservorios en las áreas verdes de la primera sección, sus desviaciones estándar e intervalos de confianza de $95 \%$ se calcularon mediante las ecuaciones 10 -12 : 


$$
\text { Estimador del total }(\hat{X})=\frac{N \bar{M}}{n \bar{M}} \sum_{i}^{n} X_{i}
$$

donde:

$N \bar{M}=$ número total de las unidades elementales en la población

$n \bar{M}=$ número total de las unidades elementales en la muestra

$X_{i}=$ valor de la característica $X$ en el conglomerado $i$

$\hat{D} \hat{E}(X)=\sqrt{\frac{N^{2}(1-f)}{n(n-1)}\left(\sum_{i}^{n} X_{i}^{2}+\hat{R}^{2} \sum_{i}^{n} M_{i}^{2}-2 \hat{R} \sum_{i}^{n} X_{i} M_{i}\right)}$

donde:

$f=$ fracción muestral $\mathrm{n} / \mathrm{N}$

$N=$ número de conglomerados en la población

$X_{i}=$ valor de la característica $\mathrm{X}$ en el conglomerado $i$

$M_{i}=$ unidades elementales en el conglomerado $i$

$\hat{R}=$ estimador de la media

Intervalo de confianza $I C(X)=\left[X \pm t_{n-1(\alpha / 2)} \hat{D} \hat{E}(X)\right]$

donde:

$X=\quad$ estimador del total o media muestral

$\hat{D} \hat{E}(\bar{X})=$ estimador de la desviación estándar de la media o total muestral

Por último, se realizó una comparación descriptiva entre el reservorio de $\mathrm{C}$ promedio estimado en este bosque urbano y los registrados en otras áreas verdes de ciudades del mundo, para analizar su almacenamiento.

\section{Resultados}

\section{Composición de especies y reservorios}

En 49.73 ha se contabilizaron 14223 individuos arbóreos pertenecientes a 102 especies y 229 árboles muertos en pie. De acuerdo con el índice de valor de importancia relativo (IVIR), F. uhdei, L. lucidum y $H$. lusitanica son las más dominantes, ya que componen cerca de $64 \%$ del arbolado y, en conjunto con $T$. mucronatum, Eucalyptus globulus, Pinus sp., E. camaldulensis, C. equisetifolia, Phoenix canariensis, Jacaranda mimosifolia, Liquidambar styraciflua, Eucalyptus sp. y Schinus molle, representan 90\% de la masa arbolada evaluada (Tabla 1). Aunque L. lucidum presentó un mayor número de árboles, F. uhdei obtuvo el porcentaje de IVIR más alto, ya que posee individuos de mayor tamaño, como lo indica su área basal. Por tanto, este índice es un indicador más robusto que la abundancia para definir la dominancia (LozadaDávila, 2010).

Las estimaciones de biomasa de la ecuación de Pillsbury et al. (1998) en ambientes urbanos (California, E.U.A.) fueron similares a los valores registrados en los árboles de F. ubdei cosechados. En contraste, la fórmula desarrollada en un bosque natural (Ares y Fownes, 2000) cuantificó 53.54\% más de biomasa que la de Pillsbury et al. (1998) en individuos con DAP mayores a $30 \mathrm{~cm}$ (Fig. 2), por lo cual en la cuantificación se empleó la de ambientes urbanos.

En la superficie inventariada se cuantificó un reservorio de 6617.4 Mg de biomasa aérea y 3067.4 Mg de C. Los valores medios por individuo arbóreo fueron de $0.458 \mathrm{Mg} \pm 0.062 \mathrm{Mg}$ de biomasa y $0.212 \mathrm{Mg} \pm 0.029 \mathrm{Mg}$ de C. Cerca de 80\% del depósito de C está consignado en los individuos de F. uhdei (29.8\%), H. lucidum (23\%), T. mucronatum (9.19\%), E. globulus (8.38\%), H. lusitanica $(5.78 \%)$ y E. camaldulensis (3.72\%). El coeficiente de correlación de Pearson entre el IVIR de cada taxón y su contribución en el reservorio de $\mathrm{C}$ fue 0.962 ( $\mathrm{p} \leq 0.001)$ e indica una alta relación positiva entre ambos. F. uhdei, T. mucronatum y las especies de eucalipto, de portes altos en su madurez (Calderón y Rzedowski, 2001; Martínez-González, 2008), contribuyen con un mayor depósito en proporción a su dominancia (IVIR). En contraste, $H$. lucidum y J. mimosifolia, de portes pequeños (Martínez-González, 2008), generaron un menor aporte con respecto a su IVIR. $H$. lusitanica fue la excepción, con una menor contribución a pesar de que puede medir hasta $30 \mathrm{~m}$ de altura (Calderón y Rzedowski, 2001), lo cual se debe a que 59.73\% de sus ejemplares eran juveniles (DAP $\leq 20 \mathrm{~cm})$. En P. canariensis, se observó un aporte de C incipiente en comparación a su IVIR (Fig. 3). 


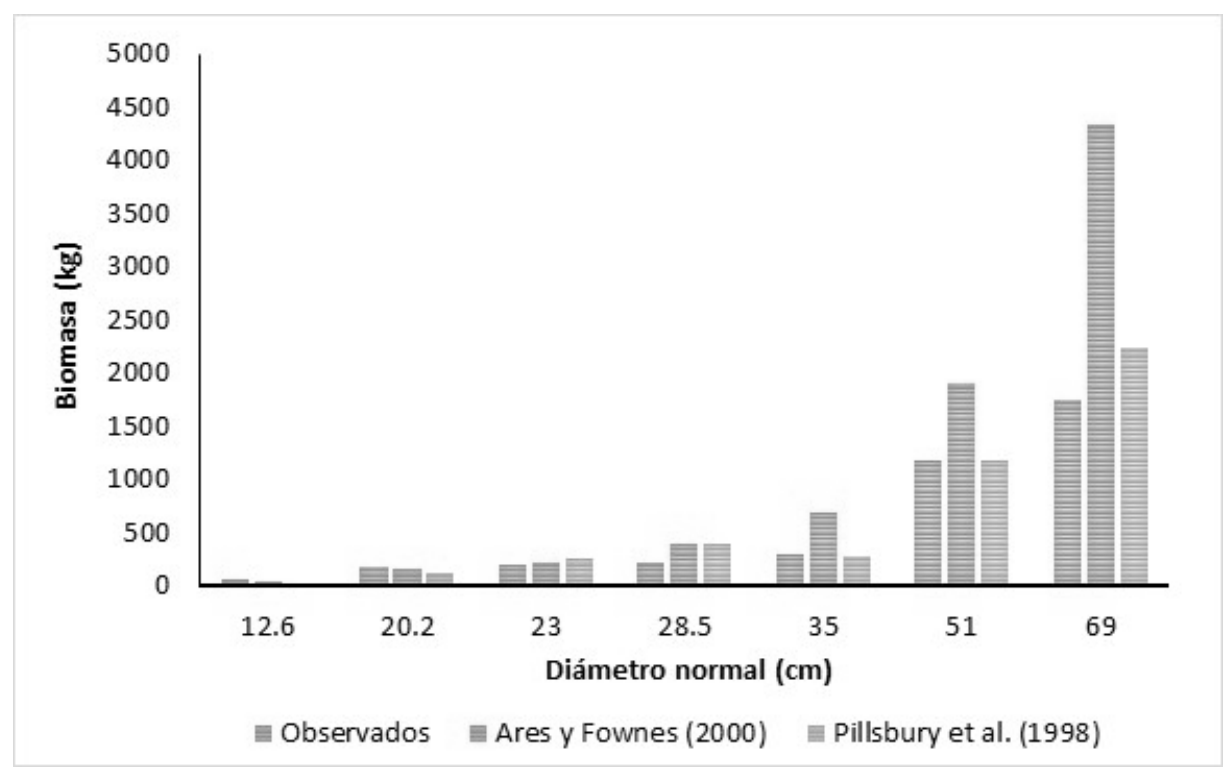

FiguRA 2. La estimación de biomasa de F. ubdei a través de ecuaciones generadas en un bosque natural (Ares y Fownes, 2000) y en uno urbano (Pillsbury et al. 1998), con los valores observados en árboles del área de estudio $(\mathrm{n}=7)$.

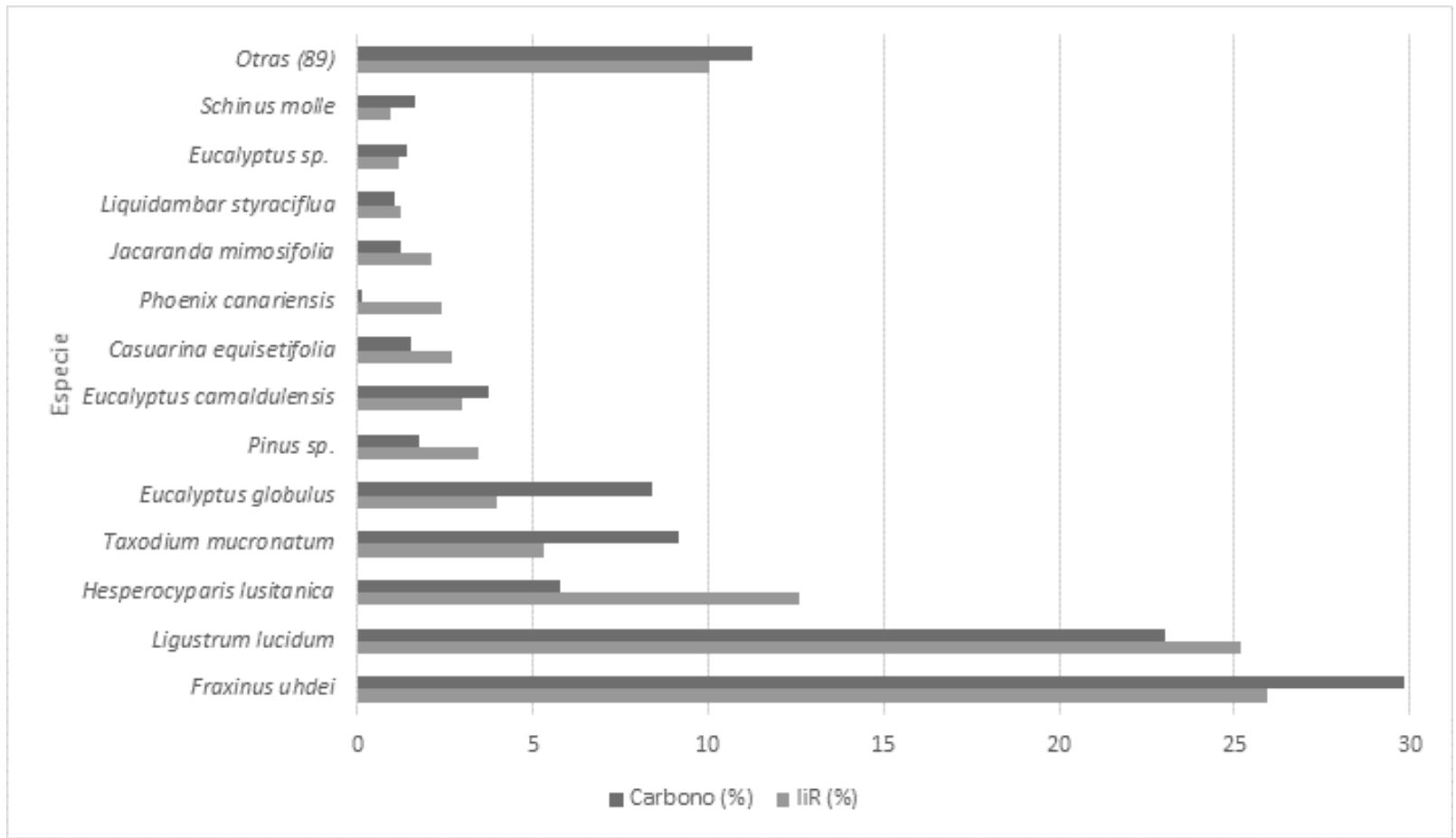

FIGURA 3. Índice de valor de importancia relativo (IVIR) y reservorio de C de las principales especies arbóreas registradas en el área de estudio. 


\section{Densidad y reservorio de carbono}

En los 11 conglomerados hubo variaciones en el depósito de C, con valores de $92.1 \mathrm{Mg} \mathrm{ha}^{-1}$ (Parque Gandhi) a $39.9 \mathrm{Mg} \mathrm{ha}^{-1}$ (Acceso Quebradora) (Tabla 2). Esto se debe a que el arbolado de Parque Gandhi presentó una gran cantidad de árboles de grandes dimensiones (DAP de hasta $148 \mathrm{~cm}$ ) y un alto valor de $\mathrm{AB}$; mientras que la segunda zona poseía en su mayoría ejemplares de J. mimosifolia y L. lucidum, con diámetros inferiores a los $82 \mathrm{~cm}$ y una menor densidad.

Se cuantificó un reservorio de $11225.7 \mathrm{Mg}$ de C en la biomasa aérea del arbolado ubicado en las áreas verdes (182 ha) de la primera sección del Bosque de Chapultepec, lo que equivale a 35 $255.3 \mathrm{Mg}$ de $\mathrm{CO}_{2}$ capturado (Tabla 3). El contenido de C promedio estimado en la primera sección (61.68 $\mathrm{Mg} \mathrm{ha}^{-1}$ ) está dentro de los intervalos registrados en bosques urbanos de otras ciudades del mundo y solamente fue menor al valor medio calculado por Nowak et al. (2013) para 28 ciudades y seis estados de EE.UU. (76.9 Mg ha-1) (Tabla 4). Esta área verde obtuvo un menor almacenamiento por unidad de superficie que la vegetación de la zona periurbana de la Ciudad de México en 1996 (80.02 $\mathrm{Mg} \mathrm{ha}^{-1}$ ), conformado por bosques de A. religiosa, Pinus sp. y Quercus sp. (Butrón-Madrigal et al. 2004) (Tabla 4).

TABLA 2. Área, número de individuos, área basal, densidad y reservorios de biomasa y C por hectárea, registrados en 11 zonas inventariadas en el área de estudio.

\begin{tabular}{|c|c|c|c|c|c|c|}
\hline Zona (conglomerado) & Área (ha) & $\begin{array}{c}\text { Número de } \\
\text { individuos por } \\
\text { hectárea }\end{array}$ & $\begin{array}{c}\text { Densidad de } \\
\text { área basal } \\
\left(m^{2} h a^{-1}\right)\end{array}$ & $\begin{array}{c}\text { Biomasa } \\
\text { (Mg) }\end{array}$ & $\begin{array}{l}\text { Carbono } \\
\text { (Mg) }\end{array}$ & $\begin{array}{l}\text { Carbono } \\
\left(\mathrm{Mg} \mathrm{ha}^{-1}\right)\end{array}$ \\
\hline Parque Tamayo** & 12.99 & 283.9 & 25.55 & 1481.4 & 693.2 & 53.3 \\
\hline Parque Gandhi** & 10.74 & 321.0 & 38.51 & 2173.0 & 1011.3 & 94.1 \\
\hline Los Pinos** & 8.48 & 506.7 & 29.96 & 1182.2 & 551.3 & 65.0 \\
\hline Jardín Botánico** & 3.81 & 167.1 & 19.96 & 333.5 & 154.0 & 40.4 \\
\hline $\begin{array}{l}\text { Histórico } \\
\text { Monumental** }\end{array}$ & 3.07 & 153.8 & 28.90 & 307.8 & 141.1 & 46.0 \\
\hline Puerta de Leones** & 2.40 & 208.1 & 26.00 & 292.9 & 129.2 & 53.8 \\
\hline $\begin{array}{ll}\text { La } & \text { Milla-Acc. } \\
\text { Chivatito* } & \end{array}$ & 2.69 & 153.6 & 19.99 & 293.7 & 133.2 & 49.4 \\
\hline Acc. Gandhi-Grutas* & 1.79 & 175.8 & 24.64 & 204.9 & 94.8 & 52.9 \\
\hline Lago Mayor** & 1.66 & 143.9 & 23.28 & 164.2 & 73.5 & 44.3 \\
\hline Parque La Amistad** & 1.61 & 234.5 & 20.10 & 142.1 & 66.2 & 41.1 \\
\hline Acc. Quebradora* & 0.48 & 283.9 & 20.62 & 41.1 & 19.1 & 39.9 \\
\hline
\end{tabular}


TABLA 3. Estimación de los reservorios y contenido promedio de biomasa y C en el área de estudio.

\begin{tabular}{clccc}
\hline \multirow{2}{*}{$\begin{array}{c}\text { Variable } \\
\end{array}$} & \multicolumn{1}{c}{ Parámetro } & Estimado & $\widehat{D} \hat{E}$ & $\begin{array}{c}\text { Intervalo de confianza } \\
\text { (95\% de confiabilidad) }\end{array}$ \\
\hline Biomasa & Contenido promedio $\left(\mathrm{Mg} \mathrm{ha}^{-1}\right)$ & 133.06 & 14.49 & $(100.78-165.34)$ \\
aérea & Reservorio $(\mathrm{Mg})$ & 24217.40 & 2423.62 & $(18817.32-29617.47)$ \\
Carbono & Contenido promedio(Mg ha-1) & 61.68 & 6.77 & (46.59- 76.76) \\
aéreo & Reservorio (Mg) & 11225.70 & 1132.37 & (8702.66-13 748.74) \\
\hline$\widehat{D} \hat{E}=$ estimador de la desviación estándar de la media muestral & & &
\end{tabular}

TABLA 4. Comparación del reservorio y contenido de C estimados en el área de estudio con los registrados en el suelo de conservación de la Ciudad de México y en bosques urbanos de ciudades del mundo.

\begin{tabular}{|c|c|c|c|c|c|}
\hline \multirow{2}{*}{ Sitio } & \multirow{2}{*}{$\begin{array}{l}\text { Área } \\
\text { (ha) }\end{array}$} & \multirow{2}{*}{$\begin{array}{l}\text { Reservorio } \\
\text { de C }(\mathrm{Mg})\end{array}$} & \multicolumn{2}{|c|}{ Contenido de C $\left(\mathrm{Mg} \mathrm{ha}^{-1}\right)$} & \multirow{2}{*}{ Referencias } \\
\hline & & & Media & Intervalo & \\
\hline $\begin{array}{lll}\text { Primera } & \text { sección } & \text { del }\end{array}$ & & & & & \\
\hline Bosque de Chapultepec, & 182.00 & 11225.8 & 61.7 & $39.9-92.1$ & Este estudio \\
\hline \multicolumn{6}{|l|}{ Ciudad de México } \\
\hline Leipzig, Alemania & 5824.00 & 352000 & 11.8 & 4.0-98.3 & $\begin{array}{l}\text { Strohbach y Haase } \\
\text { (2012) }\end{array}$ \\
\hline 28 ciudades y 6 estados, & & 643000 & & & \\
\hline & - & & 76.9 & $31.4-141.4$ & Nowak et al. (2013) \\
\hline Shenyang, China* & 0.01 & 337.0 & 33.2 & $13.1-50.1$ & Liu y Li (2012) \\
\hline $\begin{array}{l}\text { Suelo de conservación de } \\
\text { la Ciudad de México }\end{array}$ & 35485 & 3850519.9 & 80.0 & $49.3-137.7$ & $\begin{array}{l}\text { Butrón-Madrigal et } \\
\text { al. (2004) }\end{array}$ \\
\hline
\end{tabular}

\section{DISCUSIÓN}

La abundancia de especies observada es similar a la señalada por el PUEC (2002), que registró una riqueza de 110 especies en la primera sección y L. lucidum, F. uhdei y H. lusitanica fueron las más frecuentes.

La mejor estimación de la biomasa de F. uhdei, proporcionada por el modelo desarrollado en ambientes urbanos
(Pillsbury et al. 1998), con respecto al de bosques naturales (Ares y Fownes, 2000), se debe en parte a la forma de la ecuación alométrica, pues de acuerdo con Feldpausch et al. (2012), las fórmulas que solo consideran el diámetro tienden a sobrestimar la biomasa en clases diamétricas superiores, mientras que las que incluyen la altura por lo regular proporcionan estimaciones con un menor grado de error. Además, la utilización de ecuaciones 
generadas en ambientes urbanos para hacer una cuantificación más precisa de los reservorios se ha recalcado durante los últimos años (McHale et al. 2009; Aguaron y McPherson, 2012). Por ejemplo, al emplear ecuaciones de bosques naturales Nowak (1994) determinó una sobrestimación de 20\% respecto a los valores reales en 30 especies de árboles urbanos de Oak Park (Illinois, EUA). McHale et al. (2009) registraron subestimaciones de $96 \%$ y sobrestimaciones de hasta $109 \%$ en 11 especies arbóreas de Fort Collins, Colorado; que resultó en una diferencia de $60 \%$ en la cuantificación de la biomasa. Para este estudio, en seis de las 13 especies que conformaron 90\% del arbolado y en siete de los 94 taxones restantes se utilizaron ecuaciones de árboles urbanos (McPherson y Simpson, 1999; Velasco et al. 2014). Puesto que estas fórmulas se generaron con árboles urbanos de Estados Unidos., cuyas condiciones de crecimiento pueden ser similares a las del área de estudio, se espera que proporcionen una estimación aceptable. En solo 15 taxones se utilizaron ecuaciones desarrolladas en México, debido a la carencia de fórmulas.

De las especies registradas, $57.84 \%$ son exóticas, por ello se emplearon relaciones alométricas generadas en su país de origen o en EE.UU. Si bien el uso de estas ecuaciones proporciona cierta incertidumbre, se debe considerar que para las especies más frecuentes, el reservorio de $\mathrm{C}$ tuvo cierta correspondencia con el IVIR que es una medida de la dominancia de las mismas. Para mejorar las estimaciones de los reservorios de biomasa y $\mathrm{C}$, es necesario desarrollar ecuaciones con árboles urbanos, sobre todo para las que figuran entre las especies más frecuentes en el arbolado de la Ciudad de México.

Nowak et al. (2002) señalan que los árboles longevos y de grandes tallas en la madurez, tienen mayor potencial para almacenar $\mathrm{C}$ que los de corta vida y portes pequeños. Tal situación se presenta en el área de estudio y la Colonia Escandón de la Ciudad de México, donde la mayor parte del reservorio está consignado en los ejemplares de F. uhdei, H. lusitanica y $T$. mucronatum (Velasco et al. 2014). El porte y periodo de vida de las especies arbóreas son criterios adicionales a considerar en la selección, con el fin de potenciar la capacidad de captura y almacenamiento de $\mathrm{C}$, sin dejar de valorar sus otras características, como la tolerancia y capacidad de adaptación a factores adversos que les permiten sobrevivir en un ambiente urbano (Harris et al. 2004; Scharenbroch, 2012).

En congruencia con otros estudios (Nowak, 1994; Nowak et al. 2002; Liu y Li 2012; Timilsina et al. 2014), los resultados muestran que el almacén de biomasa y $\mathrm{C}$ es afectado por la estructura y composición de especies, ya que los mayores reservorios se registraron en las áreas más densas y con la mayor proporción de árboles de grandes dimensiones.

En comparación con los bosques urbanos de algunas ciudades, la primera sección constituye un reservorio de $\mathrm{C}$ importante, ya que presentó un contenido alto. Cabe aclarar que las estimaciones de estas urbes tienen una reducción de $20 \%$ de la biomasa aérea por emplear ecuaciones de bosques naturales, pero un aumento de $26 \%$ para incluir la parte subterránea (Aguaron y McPherson, 2012; Scharenbroch, 2012; Nowak et al. 2013). En el presente estudio por falta de información no se incluyó el sistema radical, aunque este componente puede representar de $16 \%$ a $41 \%$ de la parte aérea en función de las condiciones del suelo (Strohbach y Haase, 2012).

El menor almacenamiento de C por unidad de superficie estimado en esta área verde, con respecto al indicado en la vegetación de la zona periurbana de la Ciudad de México, coincide con lo registrado por Escobedo et al. (2010), quienes en un estudio realizado en Miami-Dade, Florida, registraron un mayor reservorio y capacidad de captura de $\mathrm{C}$ en el bosque periurbano, conformado por rodales de pino-encino y manglares, en comparación con el arbolado ubicado dentro de la ciudad.

El depósito de C estimado para todo el arbolado de la primera sección representa solo $0.13 \%$ del total de emisiones de gases de efecto invernadero liberados en el año 2012 en la Zona Metropolitana de la Ciudad de México (30.7 Mg de $\mathrm{CO}_{2}$ equivalente) y $0.17 \%$ de las $24.6 \mathrm{Mg}$ de $\mathrm{CO}_{2}$ equivalente del sector energético, que genera $80 \%$ de las emisiones (Velasco-Rodríguez et al., 2014). Sin embargo, el almacén de C por hectárea en la biomasa aérea del arbolado del área de estudio (226.4 $\mathrm{Mg} \mathrm{ha}^{-1} \mathrm{de}$ $\mathrm{CO}_{2}$ ) representa $92.39 \%$ de las emisiones anuales por unidad de superficie en la Colonia Escandón, Miguel Hidalgo (245 Mg ha-1 de $\mathrm{CO}_{2}$ ), que presenta altos valores de emisión de $\mathrm{CO}_{2}$ y se ubica cerca del área de estudio (Velasco et al., 2014). Tales cifras resaltan la necesidad de potenciar la captura de C del arbolado urbano mediante el mejoramiento de las áreas verdes ya existentes y la 
apertura de nuevos espacios con vegetación que contribuyan a la sustentabilidad de esta ciudad. El potencial de captura y almacenamiento de $\mathrm{C}$ se puede incrementar con un manejo adecuado de la composición de especies y el área basal del arbolado, además de proporcionar un mantenimiento adecuado que favorezca el crecimiento de los árboles (McPherson y Simpson, 1999).

A pesar de que el presente estudio provee información relevante sobre el reservorio de $\mathrm{C}$ aéreo en la primera sección y su relación con algunos componentes de la estructura y composición del arbolado, es necesario continuar los estudios que permitan cuantificar la tasa de captura de $\mathrm{CO}_{2}$, así como estimar la emisión de gases de efecto invernadero derivada de las acciones cotidianas de mantenimiento efectuadas en los árboles, para tener el balance neto de $\mathrm{C}$ y precisar la capacidad de mitigación de esta área verde.

\section{CONCLUSIONES}

En la primera sección del Bosque de Chapultepec se estimó un reservorio aéreo total de $24217 \mathrm{Mg}$ de biomasa y $11226 \mathrm{Mg}$ de C con base en el inventario de $27.3 \%$ de sus áreas verdes y el uso de relaciones alométricas. El contenido promedio de $\mathrm{C}$ en el área de estudio es relativamente alto con respecto a los registrados en bosques urbanos de otras ciudades del mundo. La ecuación de biomasa de la especie dominante, F. ubdei, desarrollada en ambientes urbanos (Pillsbury) proporcionó una mejor estimación de este parámetro que la fórmula de bosque naturales, por lo que puede emplearse para estimar el reservorio de biomasa aérea de los individuos de esta especie bajo condiciones de crecimiento similares a las del área de estudio y sin la necesidad de desarrollar una nueva ecuación. Para mejorar la estimación de los reservorios de biomasa y $\mathrm{C}$ en bosque urbanos es necesario desarrollar y/o utilizar ecuaciones de árboles urbanos.

\section{RECONOCIMIENTOS}

El presente estudio se desarrolló gracias a la información provista por el Inifap derivada del proyecto: "Diagnóstico y caracterización de la primera sección del bosque de Chapultepec", financiado por la Conafor y el Fideicomiso Pro-bosque de Chapultepec. El primer autor agradece al Conacyt la beca otorgada para la realización de sus estudios de maestría en el Colegio de Postgraduados (Colpos), Campus Montecillo.

\section{REFERENCIAS}

Acosta-Mireles, M., Vargas-Hernández, J., Velázquez-Martínez, A., \& Etchevers-Barra, J. D. (2002). Estimación de la biomasa aérea mediante al uso de relaciones alométricas en seis especies arbóreas en Oaxaca, México. Agrociencia, 36, 725-736.

Aguaron, E., \& McPherson, E. G. (2012). Comparison of methods for estimating carbon dioxide storage by Sacramento's urban forest. En L. Rattan, \& A. Bruce (Eds.), Carbon sequestration in urban ecosystems (pp. 43-70). EE. UU.: Springer. doi: 10.1007\%2F978-94-007-2366-5

Akbari, H., Davis, S., Dorsano, S., Huang, J., \& Winnett, S. (1992). Cooling our communities. A guidebook on tree planting and light-colored surfacing. EE. UU. USEPA.

Ares, A., \& Fownes, J. H. (2000). Comparisons between generalized and specific tree biomass functions as applied to tropical ash (Fraxinus uhdei). New Forests, 20, 277-286. doi: $10.1023 \% 2 \mathrm{FA} \% 3 \mathrm{~A} 1006766529550$

Benavides-Meza, H. M. (1989). Bosque urbano: la importancia de su investigación y correcto manejo. En Memoria del Congreso Forestal Mexicano 1989. Tomo II. (pp. 966-992). Estado de México, México.

Butrón-Madrigal. L., Del Valle-Cárdenas B., Escandón-Calderón, J., Gutiérrez-Guzmán, B., López-Pérez, M., MagañaRueda, V., Martínez-Gaytán, I., Medrano-García, J., Rivero-Borrel, E. E., Rodríguez-Gutiérrez, D., RosasFlores, D., Sanginés-Sayavedra, Y., Sheinbaum-Pardo, C., Trujillo-Bolio, E., \& Vázquez-Martínez, O. (2004). En O. Vázquez-Martínez (Coord.), Estrategia Local de Acción Climática del Distrito Federal. México: Secretaría del Medio Ambiente, Gobierno del Distrito Federal.

Calderón de R., G., \& Rzedowski, J. (2001). Flora Fanerogámica del $V$ alle de México (2a ed.). México: Instituto de Ecología A. C. y Comisión Nacional para el Conocimiento y Uso de la Biodiversidad.

Dobbs, C., Hernández, J., \& Escobedo, F. (2011). Above ground biomass of leaf área model based on a non-destructive method for urban trees of two communes in Central Chile. Bosque, 32(3), 287-296. doi: 10.4067/S071792002011000300010

Escobedo, F., Varela, S., Zhao, M., Wagner, J. E., \& Zipperer, W. (2010). Analyzing the efficacy of subtropical urban forest 
in offsetting carbon emissions from cities. Environmental Science \& Policy, 13, 362-372. doi: 10.1016/j.envsci.2010.03.009

Feldpausch, T. R., Lloyd, J., Lewis, S. L., Brienen, R. J. W., Gloor, M., Monteagudo-Mendoza, A., López, G. G., ..., \& Phillips, O. L. (2012). Tree height integrated into pantropical forest biomass estimates. Biogeosciences, 9, 33813403. doi: 10.5194/bg-9-3381-2012

Gobierno del Distrito Federal [GDF]. (2006). Programa de rehabilitación integral del Bosque de Chapultepec. En Secretaría del Medio Ambiente (Coord.), Memorias. Dirección General de Bosques Urbanos y Educación Ambiental (pp. 59-113), México: GDF.

Harris W. R., Clark, R. J., \& Matheny, P. N. (2004). Arboriculture: integrated management of landscape trees, shrubs, and vines (4a ed.). EE. UU.: Prentice Hall.

Heisler, G. M. (1986). Energy saving with trees. Journal of Arboriculture, 12(5), 113-125.

Intergovernmental Panel on Climate Change [IPCC]. (2006). Agricultura, silvicultura y otros usos de la tierra (vol. 4). En IPCC. Directrices del IPCC de 2006 para los inventarios nacionales de gases de efecto invernadero (pp. 1-93). Japón: IPCC.

Jenkins, J. C., Chojnacky, D.C., Heath, L. S., \& Birdsey, R. A. (2003). National-scale biomass estimators for United States Tree Species. Forest Science, 49(1), 12-35.

Liu, C., \& Li, X. (2012). Carbon storage and sequestration by urban forests in Shenyang, China. Urban Forestry \& Urban Greening, 11, 121-128. doi: 10.1016/j.ufug.2011.03.002

Livesley, S. J., McPherson, E. G., \& Calfapietra, C. (2016). The urban forest and ecosystem services: impacts on urban water, heat and pollution cycles at the trees, street and city scale. Journal of Environmental Quality, 45, 119-124. doi: 10.2134/jeq2015.11.0567

López-López, S. F., Martínez-Trinidad, T., Benavides-Meza, H., García-Nieto, M., \& de los Santos-Posadas, H. M. (2017). Non-destructive method for above-ground biomass estimation of Fraxinus ubdei (Wenz.) Lingelsh in an urban forest. Urban Forestry \& Urban Greening, 24, 62-70. doi: 10.1016/j.ufug.2017.03.025

Lozada-Dávila, J. R. (2010). Consideraciones metodológicas sobre los estudios de comunidades forestales. Revista Forestal Venezolana, 54(1), 77-88.

Martínez-González, L. (2008). Árboles y áreas verdes urbanas de la Ciudad de México y su zona metropolitana. México: Fundación Xochitla.
McHale, M. R., McPherson, E. G., \& Burke, I. C. (2007). The potential of urban tree plantings to be cost effective in carbon credit markets. Urban Forestry \& Urban Greening, 6, 49-60. doi:10.1016/j.ufug.2007.01.001

McHale M. R., Burke, I. C., Lefsky, M. A., Pepper, P. J., \& McPherson, E. G. (2009). Urban forest biomass estimates: is it important to use relationships developed specifically for urban trees? Urban Ecosystems, 12, 95-113. doi: 10.1007\%2Fs11252-009-0081-3

McPherson, E. G., \& Simpson, J. R. (1999). Carbon dioxide reduction through urban forestry: Guidelines for professional and volunteer tree planters. Gen. Tech. Rep. PSW-GTR-171. EE. UU.: USDA Forest Service, Pacific Southwest Research Station.

McPherson, E. G., Simpson, J. R., Peper, P. J., \& Aguaron, E. (2008). Urban forestry and climate change. EE. UU.: USDA Forest Service, Pacific Southwest Research Station.

Nowak, D. J. (1994). Atmospheric dioxide carbon reduction by Chicago's urban forest. En E. G. McPherson, D. J. Nowak, \& R. A. Rowntree, (Eds.), Chicago's urban forest ecosystem: results of the Chicago Urban Forest Climate Project. (General Technical Report NE-186). EE. UU.: USDA Forest Service, Northeastern Forest Experiment Station.

Nowak, D. J., Stevens, J. C., Sisinni, S. M., \& Luley, C. J. (2002). Effects of urban tree management and species selection on atmospheric carbon dioxide. Journal of Arboriculture, 28(3), 113-122.

Nowak, D. J., Greenfield, E. J., Hoehn, R. E., \& Lapoint, E. (2013). Carbon storage and sequestration by trees in urban and community areas of the United States. Environmental Pollution, 178, 229-236. doi: 10.1016/j.envpol.2013.03.019

Pearson, T., Brown, S., \& Birdsey, R. (2007). Measurement guidelines for the sequestration of forest carbon. (General Technical Report NRS-18). EE. UU.: USDA Forest Service, Northeastern Forest Experiment Station.

Pillsbury, N. H., Reimer, J. L., \& Thompson, R. P. (1998). Tree volume equations for fifteen urban species in California. (General Technical Report 7). EE. UU.: California Polytechnic State University, Urban Forest Ecosystem Institute.

Programa Universitario de Estudios sobre la Ciudad [PUEC]. (2002). Proyecto ejecutivo sobre la implementación del manejo integral y desarrollo autosostenible del Bosque de Chapultepec. México: Universidad Nacional Autónoma de México.

Rügnitz-Tito, M., Chacón-León, M., \& Porro, R. (2008). Guía para la determinación de carbono en pequeñas propiedades rurales. Brasil: 
Consorcio Iniciativa Amazónica (IA) y Centro Mundial Agroforestal (ICRAF).

Scharenbroch, B. C. (2012). Urban trees for carbon sequestration. En L. Rattan, \& A. Bruce (Eds.), Carbon sequestration in urban ecosystems (pp. 121-138). EE. UU.: Springer.

Strohbach, M. W., \& Haase, D. (2012). Above-ground carbon storage by urban trees in Leipzig, Germany: Analysis of patterns in a European city. Landscape Urban Plan, 104, 95104. doi: 10.1016/j.landurbplan.2011.10.001

Timilsina, N., Escobedo, F. J., Straudhammer, C. L., \& Brandeis, T. (2014). Analyzing the causal factors of carbon stores in a subtropical urban forest. Ecological Complexity, 20, 23-32. doi: 10.1016/j.ecocom.2014.07.001

Velasco, E., Perrusquia, R., Jiménez, E., Hernández, F., Camacho, P., Rodríguez, S., Retama, A., \& Molina, L. T. (2014). Sources and sinks of carbon dioxide in a neighborhood of Mexico City. Atmospheric Environment, 97, 226-238. doi: 10.1016/j.atmosenv.2014.08.018

Velasco-Rodríguez, G., Becerra-Delgado, M. L., Vázquez Ramírez, I., Skwierinski-Durán, A., Haro González, M. E., \& Ortega-Ramírez, M. (2014). Estrategia Local de Acción Climática: Ciudad de México 2014-2020. México: Centro
Mario Molina y Secretaría del Medio Ambiente del Distrito Federal.

Zarco-Espinosa, V. M., Valdez-Hernández, J. L., Ángeles-Pérez, G., \& Castillo-Acosta, O. (2010). Estructura y diversidad de la vegetación arbórea del Parque Estatal Agua Blanca, Macuspana, Tabasco. Universidad y Ciencia, 26(1), 1-17.

Manuscrito recibido el 22 de agosto de 2017

Aceptado el 28 de febrero de 2018

Publicado el 15 de octubre de 2018

Este documento se debe citar como:

López-López, S. F., Martínez-Trinidad, T., Benavides-Meza, H. M., García-Nieto, M., \& Ángeles-Pérez, G. (2018). Reservorios de biomasa y carbono en el arbolado de la primera sección del Bosque de Chapultepec, Ciudad de México. Madera y Bosques, 24(3), e2431620. doi: 10.21829/myb.2018.2431620

Madera y Bosques por Instituto de Ecología, A.C. se distribuye bajo una Licencia Creative Commons Atribución-NoComercialCompartirlgual 4.0 Internacional. 